\title{
Nasal Obstruction
}

National Cancer Institute

\section{Source}

National Cancer Institute. Nasal Obstruction. NCI Thesaurus. Code C50662.

Blockage of the nasal passages. 